\title{
Identificação de áreas prioritárias para conservação da avifauna na bacia hidrográfica do rio una, Ibiuna/SP
}

A fragmentação de habitats e o isolamento de populações é uma das ameaças mais sérias à biodiversidade. Assim, o presente estudo teve como objetivo desenvolver uma metodologia de identificação de áreas de risco à biodiversidade utilizando a avifauna e técnicas de geoprocessamento. Para isso, foi gerado o Indicador de Exposição Antrópica (IEA) a partir do Índice de Circularidade (IC) e Índice de Efeito de Borda (IEB), além da utilização dos Índice de Vegetação por Diferença Normalizada (NDVI), Densidade de drenagem e indicadores da abundância e riquiza da avifauna para a análise de fragmentos florestais. Os resultados de IC e IEB mostraram que os fragmentos possuem, em sua maioria, forma muito alongada e pressão antrópica moderada. O inventário da avifauna mostrou 147 espécies, com abundância de 9025 indivíduos. Desta forma, com o cruzamento das variáveis, foi obtido o mapa de risco da avifauna, identificando os fragmentos 12 e 15 como de menor risco à perda da biodiversidade. Com relação às áreas de alto risco, foram identificados os fragmentos 4,5 e 7. Esses fragmentos possuem baixa qualidade vegetal, baixa biodiversidade além de sofrer grande pressão antrópica. $O$ entorno desses fragmentos é composto por áreas de agricultura, pastagens e edificações rurais, o que pode acelerar o processo de perda da biodiversidade. Os demais fragmentos foram classificados como de médio risco. Por fim, este trabalho permitiu classificar a atual situação de fragmentos florestais na bacia do Rio Una, auxiliando os gestores públicos na tomada de decisão e planejamento ambiental sustentável destes fragmentos.

Palavras-chave: Geoprocessamento; Indicadores Ambientais; Biodiversidade; Avifauna.

\section{Identification of priority risk areas for avifaunal conservation in una river watershed, Ibiúna/SP}

\begin{abstract}
Habitat fragmentation and populational isolation is one of the most serious biodiversity's threats. Thus, the present study aimed to develop a methodology for the identification of biodiversity risk areas using avifauna and geoprocessing techniques. For this, the Anthropic Exposure Indicator (IEA) was generated from the Circularity Index (IC), the Edge Effect Index (IEB), besides the use of Normalized Difference Vegetation Index (NDVI), Density of drainage and indicators of the abundance and richness of the avifauna to analyze forest fragments. The IC and IEB results showed that the fragments have, in their majority, very elongated form and moderate anthropic pressure. The avifauna inventory showed 147 species, with an abundance of 9025 individuals. Therefore, with the crossing of the variables, the risk map of the avifauna was obtained, identifying that the fragments 12 and 15 were classified as lower risk to biodiversity loss. Regarding the high risk areas, the fragments 4, 5 and 7 were identified. These fragments have low vegetal quality, low biodiversity and great influence of anthropic pressure. The surroundings of these areas are composed of agricultural areas, pastures and rural buildings which can accelerate the process of biodiversity loss. The remaining forest fragments are classified as medium risk. Finally, this work allowed to classify the current situation of forest fragments in the Una River watershed, assisting public managers in the decision making and sustainable environmental planning of these fragments.
\end{abstract}

Keywords: Geoprocessing; Environmental Indicators; Biodiversity; Birdlife.

\section{Topic: Engenharia Ambiental}

Reviewed anonymously in the process of blind peer

Jomil Costa Abreu Sales

Universidade Estadual Paulista Júlio de Mesquita Filho, Brasil http://lattes.cnpq.br/0266681173969604

jomilc@gmail.com

Darllan Collins da Cunha e Silva

Universidade Estadual Paulista Júlio de Mesquita Filho, Brasil http://lattes.cnpq.br/1323110670876692

darllanamb@yahoo.com.br

\section{Roberto Wagner Lourenço}

Universidade Estadual Paulista Júlio de Mesquita Filho, Brasil http://lattes.cnpq.br/8484129953169399

robertoww@sorocaba.unesp.br

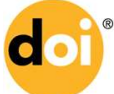

DOI: 10.6008/SPC2179-6858.2017.004.0011
Received: $\mathbf{1 3 / 0 7 / 2 0 1 7}$

Approved: 13/10/2017
Referencing this:

SALES, J. C. A.; SILVA, D. C. C.; LOURENÇO, R. W.. Identificação de áreas prioritárias para conservação da avifauna na bacia hidrográfica do rio una, Ibiúna/SP. Revista Ibero-Americana de Ciências Ambientais, v.8, n.4, p.128-142, 2017. DOI: http://doi.org/10.6008/SPC2179-6858.2017.004.0011 


\section{INTRODUÇÃO}

Embora a fragmentação dos remanescentes florestais seja um fenômeno recorrente em todo o mundo, em florestas tropicais isso tem sido mais recorrente, levando grande parte da vasta biodiversidade das florestas a se tornarem ameaçadas de extinção (CANALE et al., 2012). Uma das consequências da fragmentação é um fenômeno também conhecido como efeito de borda.

O efeito de borda, em longo prazo, antecipa a extinção local em decorrência da fragmentação e isolamento dos remanescentes florestais. Afeta as populações locais das espécies da fauna e flora, acarretando em mudanças nos processos ecológicos, como a polinização, predação, comportamento territorial e hábitos alimentares especialmente de aves dispersoras de grandes sementes e mamíferos de grande porte, vulneráveis à perda de hábitat e ao risco de extinção local (CORRÊA, 2011; LIAO, 2013; SALES et al., 2016).

No caso específico da avifauna, que constitui o grupo dos vertebrados mais estudados e conhecidos em razão dos hábitos predominantemente diurnos e do padrão conspícuo de cores, são de extrema importância para o controle biológico, dispersão de sementes e na verificação das condições ecológicas de um determinado ambiente, atuando como bioindicadores e servindo de base para planos de manejo de áreas de proteção (SILVEIRA-NETO et al., 1995; NIEMI et al., 2004; PENSE et al., 2005; PIRATELLI et al., 2008; ABREU et al., 2009).

Entretanto, a maioria dos estudos relacionados à resposta das populações e comunidades de aves brasileiras sob as ações de fragmentação das florestas têm sido centrado nas mudanças associadas ao desenvolvimento urbano em contraste com os ambientes de floresta. Estudos que considerem a instabilidade ambiental criada pelo desmatamento, e consequente desaparecimento da avifauna, têm sido pouco desenvolvidos (AMBUEL et al., 1983; DÁRIO et al., 2000; DÁRIO et al., 2002; ALEIXO et al., 2007; FERGER et al., 2014).

Nesse cenário, o desenvolvimento de indicadores ecológicos para detectar mudanças no meio ambiente pode oferecer grande colaboração no entendimento dos efeitos da fragmentação da paisagem, no empobrecimento e perda substancial de espécies endêmicas de aves, principalmente as com exigências ecológicas bem definidas (PIRATELLI et al., 2008; LOURENÇO et al., 2014).

Além disso, a interação desses indicadores com elementos espaciais ganham cada vez mais atenção, à medida que ajudam a compreender a estrutura complexas e a forma como essa influência determinadas relações ecológicas, ou seja, o fluxo de energia, materiais e espécies entre os componentes ecossistêmicos (FORMAN et al., 1992; BUREL et al., 2002; MARENZI et al., 2005; NIEMI et al., 2004; WATRIN et al., 2005; PIRATELLI et al., 2008).

O estudo de indicadores ambientais, baseado na hipótese da interação entre os componentes bióticos e abióticos, medidos espacialmente, podem promover a solução de um dos principais desafios ambientais da atualidade, como por exemplo, a proteção dos componentes da paisagem e conservação da biodiversidade (BUREL et al., 2002; FORMAN et al., 1992; MARENZI et al., 2005; CARDOSO et al., 2010; SANTOS et al., 2011; LOURENÇO et al., 2015; SILVA et al., 2016). 
Assim, este artigo apresenta os resultados de uma pesquisa que propõe uma metodologia baseada em indicadores ambientais que visam identificar áreas de risco e prioritárias para a conservação e manutenção de habitats. Para isso, foram estudados indicadores da ecologia da paisagem, da riqueza e abundância da avifauna com o uso de técnicas de geoprocessamento e modelamento matemático como forma de interação dos indicadores com base no grau de correlação da exposição antrópica dos fragmentos florestais na paisagem local.

\section{MATERIAIS E MÉTODOS}

\section{Área de estudo}

Os dados referentes a este trabalho foram obtidos em trabalhos de campo realizados na Bacia Hidrográfica do Rio Una, localizada no Estado de São Paulo, Brasil, conforme se ilustra na figura 1. Visto que é um dos mais importantes mananciais de captação de água da região, a área de estudo possui significativa importância, uma vez que abastece uma população estimada em mais de 1,5mi de pessoas, além de conter extensão faixas de matas nativas e estágios diversificados de regeneração (CAVANI et al., 2008; SILVA, et al. 2016).

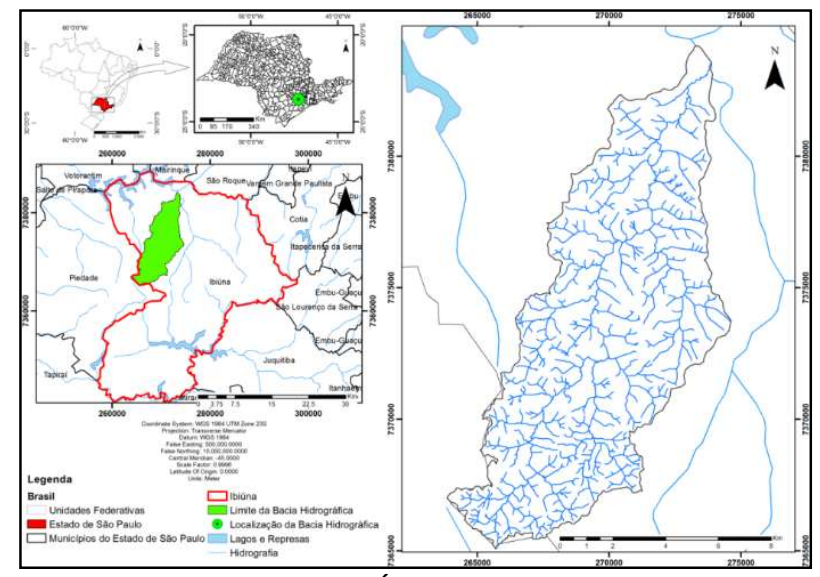

Figura 1: Área de estudo

\section{Obtenção e tratamento dos dados}

Foi elaborada uma base cartográfica digital contendo informações relevantes sobre os limites da área de estudo, rede hidrográfica, curvas de nível e pontos cotados e demais informações relevantes que serviu de para o reconhecimento cartográfico da área. O mapeamento do uso do solo e cobertura vegetal foi realizado a partir da interpretação das imagens de satélite multiespectrais ortorretificadas do sensor SPOT 5 (Satellite Pour l'Observation de la Terre) com resolução espacial de 2,5m do ano de 2010 em composição cor verdadeira (CPLA/SEMA, 2010) feito com o auxílio do GIS ArcMap 10.0 (ESRI, 2010).

Foi empregado o método de classificação supervisionada multivariada de Máxima Verossimilhança e classes de Solo exposto, Pastagem, Matas, Ecossistemas Aquáticos, Edificações Urbanas, Edificações Rurais, Campos Sujos, Agriculturas de longo período e Agricultura de curto período identificado. Campanhas de campo foram realizadas para retificação e confirmação de reconhecimento dos usos (SALES et al., 2016). 
Após o mapeamento da cobertura da terra, os fragmentos florestais foram segmentados e selecionados de acordo com a sua localização geográfica, tamanho e composição vegetal. Nesta etapa foi utilizado o Índice de Vegetação por Diferença Normalizada (NDVI) para identificar os fragmentos com características florestais mais homogêneas e de maior vigor vegetativo.

O NDVI foi elaborado a partir das bandas do infravermelho próximo e do vermelho segundo a fórmula: NDVI = (NIR-VIS) / (NIR+VIS), onde: NDVI = Índice de Vegetação por Diferença Normalizada; NIR = Banda do infravermelho próximo do sensor SPOT 5; e VIS = Banda do vermelho no comprimento do visível do sensor SPOT 5.

Foram selecionados 15 fragmentos com área superior a 10 hectares para levantamento de avifauna, considerando-se a homogeneidade florestal detectada no mapa de NDVI, a distribuição dos níveis altimétricos e declividades, subprodutos do mapa de relevo, e de acordo com o perfil topográfico longitudinal elaborado a partir das curvas de nível.

\section{Índice de Circularidade (IC)}

Em seguida foi construído um indicador de circularidade (IC) para os fragmentos florestais selecionados. Segundo Viana et al. (1998), o IC é utilizado para demonstrar o potencial de interferência de seu entorno pela característica da forma circular ou alongada do fragmento. Nesse índice não é considerado a importância ecológica do fragmento, apenas classifica-o quanto a sua geometria. Os valores de IC são gerados em um intervalo entre 0 e 1 , sendo os valores próximos de 1 indicam fragmentos com tendência a forma circular, e os valores próximos de 0 indicam fragmentos com tendência a forma alongada conforme a fórmula: IC $=\frac{2 \sqrt{\pi \mathrm{A}}}{\mathrm{P}}$, na qual: $\mathrm{IC}=$ Índice de Circularidade; $\mathrm{A}=$ Área do fragmento; e $\mathrm{P}=$ Perímetro do fragmento.

\section{Índice de Efeito de Borda (IEB)}

Ainda, para avaliar o efeito causado pelo tipo de atividade antrópica presente no entorno dos fragmentos, foi gerado o Índice de Efeito de Borda (IEB). O índice foi criado com base na medida da superfície de contato imediato dos diferentes tipos de uso do solo com o perímetro dos fragmentos florestais, atribuindo-se pesos de acordo com seu grau de importância considerando-se as 9 classes de uso do solo conforme adaptada de Lourenço et al. (2014).

Tabela 1: Valores atribuídos às classes de uso do solo.

\begin{tabular}{|l|l|}
\hline \multicolumn{1}{|c|}{ Uso do Solo } & Valores \\
\hline Matas & 0 \\
\hline Ecossistemas Aquáticos & 0 \\
\hline Campos Sujos & 0,15 \\
\hline Agricultura de longo período & 0,35 \\
\hline Pastagem & 0,45 \\
\hline Agricultura de curto período & 0,60 \\
\hline Solo exposto & 0,75 \\
\hline Edificações Rurais & \\
\hline Edificações Urbanas & \\
\hline
\end{tabular}




\section{Indicador de Exposição Antrópica (IEA)}

O Indicador de Exposição Antrópica (IEA) foi elaborado a partir dos valores do Índice de Circularidade (IC) e dos valores do Índice de Efeito de Borda (IEB). A esses índices, foram estipulados pesos referentes à importância de cada um no fator de sustentabilidade dos fragmentos frente à exposição às atividades antrópicas desenvolvidas no seu entorno.

O IC recebeu peso 1, enquanto que o IEB recebeu peso 2 . Como o valor de IEB varia de 0 a 1 sendo 0 a condição de maior proteção, foi subtraído 1 do valor do IEB. Assim o IEA com variação de 0 a 1 , sendo 0 o cenário de maior exposição aos efeitos das atividades antrópicas sobre o fragmento e 1 o cenário de menor pressão das atividades antrópicas (LOURENÇO et al., 2014). Portanto, o IEA é a média ponderada dos valores do IC e IEB como mostrado na fórmula: IEA = IC+2 (1-IEB)/3, onde: IC = Índice de Circularidade; IEB = Índice de Efeito de Borda; e IEA = Indicador de Exposição Antrópica.

\section{Densidade de Drenagem (Dd)}

Foi realizado nos fragmentos para identificar a presença ou ausência de cursos d'água o cálculo da densidade de drenagem (Dd) com o auxílio do software ArcMap 10.0. Segundo Cardoso (2006), a Dd reflete a influência das características topográficas, litológicas, pedológicas e da cobertura vegetal e incorpora a influência antrópica dos fragmentos. A Dd tem uma influência significativa e é calculada pela divisão do Comprimento total de todos os segmentos $(L)$ pela área dos fragmentos em quilômetros (A), conforme a fórmula: $\mathrm{Dd}=\mathrm{L} / \mathrm{A}$, na qual: $\mathrm{Dd}=$ Densidade de drenagem; $\mathrm{L}=$ Comprimento total dos canais; e $\mathrm{A}=$ Área da bacia.

\section{Diversidade e riqueza das espécies da avifauna}

O levantamento da diversidade e riqueza das espécies da avifauna foi realizado pelo método clássico de observação direta. As observações das espécies foram realizadas por meio de caminhadas pelas trilhas já existentes nos fragmentos florestais e em pontos estratégicos, com auxílio de dois binóculos um com lentes de $50 \mathrm{~mm}$ e potencial de aumento de $4 x$, e outro de $50 \mathrm{~mm}$ com aumento de $10 x$ e zoom ótico de 30x e um sistema de alto-falante portátil para atrair as aves. Para a identificação das espécies, foram utilizados os Guias de Campo Aves da Grande São Paulo (BECKER et al., 1991; SIGRIST, 2013). Foram realizadas quatro repetições amostrais de 8 horas nos horários matutino e vespertino em cada uma dos fragmentos nas quatro primeiras horas do dia e quatro horas no período da tarde encerrando sempre com o pôr-do-sol nos meses de verão e primavera (BECKER et al., 1991; DEVELEY et al., 2006; CARVALHO-JÚNIOR et al., 2008).

\section{Análise da população de Avifauna}

Para estimar a biodiversidade com base na distribuição numérica dos indivíduos das espécies em função do número total de individuos existentes em cada amostra, foram utilizadas medidas baseadas na riqueza de espécies, como o cálculo da densidade da população de avifauna, conforme a fórmula: $\mathrm{D}=\frac{\mathrm{S}_{\text {frag }}}{\Delta_{\text {frag }}}$, 
onde: $D=$ Densidade populacional; $S$ frag= Número total de indivívuos por fragmento; e $\Delta$ frag= Área do fragmento.

Também foi calculada a biodiversidade pelo Índice de Riqueza de Menhinick (DMn). Trata-se de um índice que leva em conta o número de espécies (s) e a raiz quadrada do número total de indivíduos (BARROS, 2007). Estes índices combinam o número de espécies registrado (S) com o número total de indivíduos (N) calculado pela fórmula: $D_{M n}=S / \sqrt{N}$, onde: $\mathrm{D}_{\mathrm{Mn}}=$ Índice de Riqueza de Menhinick; $\mathrm{S}=$ Número de espécies amostradas; N= é o número total de indivíduos em todas as espécies.

Outra análise foi a de riqueza e equabilidade pelo Índice de Shannon e de Simpson, respectivamente. Também chamado de Índice de Diversidade de Shanon-Wiener $\left(H^{\prime}\right)$ é uma análise apropriada para amostras aleatórias de espécies de uma comunidade, e foi estimado através da fórmula: $H^{\prime}=\sum_{i=1}^{S} P_{i} \operatorname{Ln} P_{i}$, onde: $\mathrm{H}^{\prime}=$ Índice de Diversidade; S= Número de espécies amostradas; e Pi= Proporção da espécie em relação ao número total de espécies amostradas.

\section{Identificação do nível de risco e das áreas prioritárias para conservação}

Portanto, para determinação das áreas de risco e prioritárias para a conservação da avifauna foram utilizados os valores do IEA, a média dos valores de NDVI e os índices de diversidade de Menhinick e ShannonWiener e Simpson para comporem a equação: $A_{\text {prio }}=\operatorname{IEA} \times \mu_{\mathrm{NDVI}} \times \mu_{\mathrm{DMn}} \times \mu_{\mathrm{H}^{\prime}}$,onde: $A_{\text {prio }}=$ Áreas prioritárias para conservação; IEA = Índice de Exposição Antrópica; $\mu_{\mathrm{NDVI}}=$ Média de NDVI; $\mu_{\mathrm{Dmn}}=$ Média do Índice de Riqueza de Menhinick; e $\mu_{H^{\prime}}=$ Média do Índice de Diversidade.

Os fragmentos foram classificados de 1 a 5 , sendo os valores entre 0 e 2 , foram identificados como áreas de maior impacto e classificados como de Alto Risco, enquanto que os fragmentos com valores maiores ou igual a 4 foram classificados como de Baixo Risco, portanto os valores intermediários são classificados como de Médio Risco. Após a identificação das áreas de risco, foi gerado um mapa, indicando as áreas prioritárias para as ações de proteção e conservação.

\section{RESULTADOS E DISCUSSÃO}

\section{Elementos de caracterização geomorfométricas}

A partir das informações referentes à altimetria e densidade de drenagem foi realizada a compartimentação morfométrica da Bacia Hidrográfica do Rio Una, conforme descrito na tabela 2. Segundo o critério utilizado para compartimentação altimétrica da área de estudo, a porção do Alto Una apresentou a maior concentração (densidade) de drenagem $(3,5)$ seguidos do Médio e Baixo Una, com 2,8 e 2,5 respectivamente. Isso se deve ao fato de o Alto Una estar nas cabeceiras e possuir uma grande quantidade de nascentes, característico desta área.

Tabela 2: Densidade de drenagem em função da variação altimétrica, comprimento e área dos cursos d'água.

\begin{tabular}{|c|c|c|c|c|}
\hline $\begin{array}{c}\text { Divisão da Bacia } \\
\text { hidrográfica do Una }\end{array}$ & $\begin{array}{c}\text { Variação altimétrica (h) } \\
\text { em metros }\end{array}$ & $\begin{array}{c}\text { Comprimento total dos cursos de } \\
\text { água (Lt) em } \mathbf{~ k m}\end{array}$ & $\begin{array}{c}\text { Área total (A) } \\
\mathbf{e m ~ k m}^{\mathbf{2}}\end{array}$ & $\begin{array}{c}\text { Densidade de } \\
\text { Drenagem (Dd) }\end{array}$ \\
\hline Baixo Una & $836-850$ & 54,38 & 22,07 & 2,5 \\
\hline Médio Una & $850-940$ & 150,17 & 53,84 & 2,8 \\
\hline Alto Una & $940-1175$ & 72,79 & 20,51 & 3,5 \\
\hline
\end{tabular}




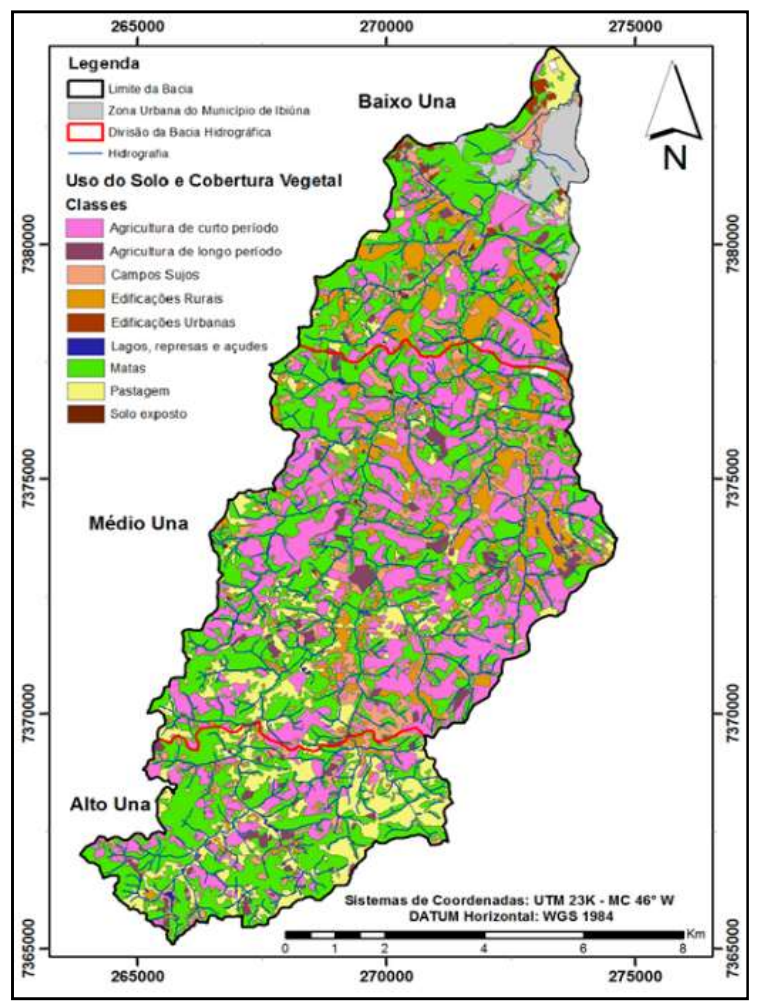

Figura 2: Mapa de uso do solo e cobertura vegetal da Bacia Hidrográfica do Rio Una. Mostra a distribuição dos diferentes tipos de uso, sendo possível observar a forte influência das atividades antrópicas nas três regiões da bacia, que deve contribuir negativamente para a biodiversidade da avifauna.

Considerando-se o período em análise, foi verificado que a classe que apresentou maior ocorrência em área foi a de Matas para as três compartimentações analisadas, Baixa, Médio e Alto Una. É importante destacar que parte significativa da principal aglomeração urbana essa localizada na porção Nordeste do Baixo Una, além da presença de agricultura de curto período e campos sujos. Trata-se de uma área de grande impacto negativo sobre os fragmentos florestais que ali se encontram. A área do Médio Una apresenta 30\% de sua extensão coberta por áreas de agricultura de curto período, além de edificações rurais e campos sujos. Por fim, o Alto Una, é predominado por áreas de pastagens, agricultura de longo e curto período.

Em seguida ,foram selecionados 15 fragmentos florestais para avaliar o IC, IEB e Densidade de drenagem (Dd) além do inventário da avifauna. Essa seleção foi feita tendo como base o Mapa de NDVI sobreposto ao uso da terra conforme pode ser visto nas figuras 3,4 e 5 , que mostram os fragmentos selecionados em seus respectivos compartimentos morfológicos, Baixo, Médio e Alto Una.

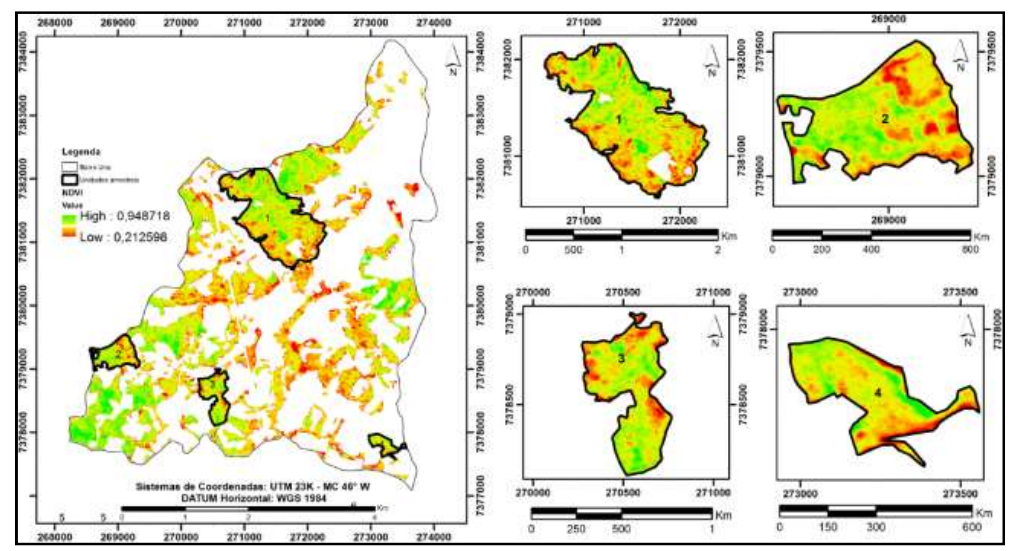

Figura 3: NDVI do Baixo Una e suas respectivas unidades amostrais. 


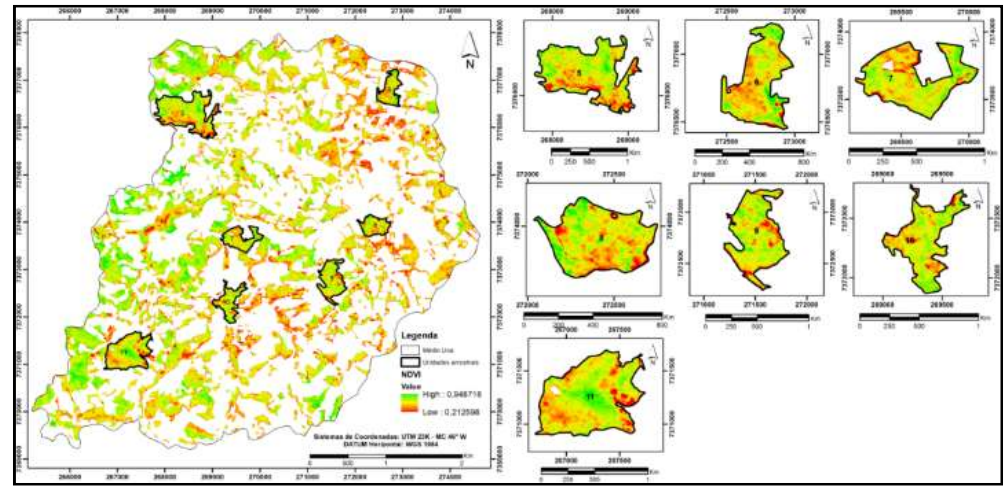

Figura 4: NDVI do Médio Una e suas respectivas unidades amostrais.

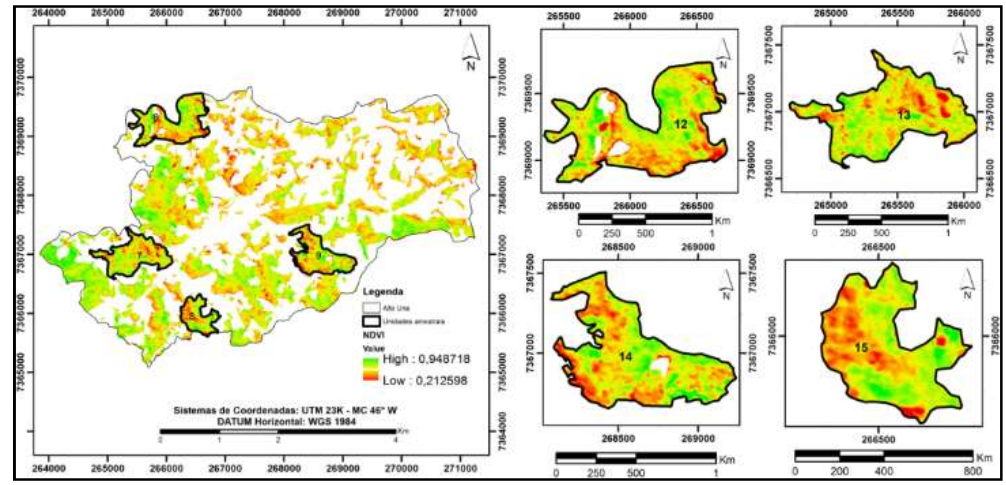

Figura 5: NDVI do Alto Una e suas respectivas unidades amostrais.

O índice de circularidade determina a tendência em relação à forma de um fragmento. A importância dessa análise para os estudos de dinâmica e estruturas de fragmentos florestais evidencia-se na possibilidade de indicar o nível de proteção de seu interior em relação ao efeito de borda. Como resultado da análise de IC, de acordo com Viana et al. (1998), os fragmentos 8, 11 e 15, possuem forma classificada como alongada sendo os fragmentos 8 e 11 pertencentes ao Médio Una e o fragmento 15 ao Alto Una.

Os demais fragmentos florestais apresentam IC menor que 0,6, sendo classificados como muito alongados, demonstrando a fragilidade dos fragmentos florestais. A partir dos resultados de IEB, foi possível classificar os fragmentos 3, 10 e 12 como sendo de Baixa Pressão Antrópica, os demais fragmentos foram classificados como de Média Pressão Antrópica, não havendo nenhum fragmento sob alta pressão antrópica, ou sob situação extrema de isolamento.

\section{Diversidade de dominância das espécies}

Quanto ao inventário da avifauna, foram observadas 147 espécies de 43 Famílias e 18 Ordens, de um universo amostral de 9025 indivíduos. Dentre as famílias, a que teve o maior número de espécies avistadas foi a TYRANNIDAE com 23 espécies, com destaque para as Pitangus sulphuratus (Bem-te-vi), Tyrannus melancholicus (Suiriri) e Tyrannus savana (Tesoura), encontradas em ambientes diversos, como campos abertos, áreas mais urbanizadas e borda de matas. Uma das principais características da família tyrannidae é o forrageamento, alimentam-se essencialmente de insetos, predominantemente de artrópodes, mas algumas espécies são frugívoras, granívoras e carnívoras. 
Segundo Sigrist (2013), as três espécies predominantes se caracterizam por serem espécies pouco exigentes, conseguem se adaptar muito bem em uma variedade de ambientes, por exemplo, bordas de matas, clareiras de florestas, capoeiras, plantações, eucaliptais, cerrados, caatinga, pastos sujos, mangues, parques e ambientes antropizados.

Os representantes da família tyrannidae, são geralmente insetívoros, mas apesar disso, algumas espécies como Pitangus sulphuratus são oportunistas e se alimentam também de peixes, ovos e até mesmo restos de alimentos e dessa forma conseguem se adaptar em qualquer ambiente, diferentemente das outras espécies que são mais especialistas, portanto, mais dependentes de ambientes abundantes em insetos (Sick, 2001).

A segunda família mais avistada foi a thraupidae, com o avistamento de 10 espécies diferentes. Esta família representa um vasto grupo composto principalmente de aves de copa, que ocorrem em bordas de florestas e áreas semiabertas, com forrageamento em grupo e hábitos onívoros. Portanto, trata-se também de uma família com características generalistas, pouco exigentes com relação aos seus habitats (Sigrist, 2009).

As espécies avistadas com maior frequência foram Thraupis sayaca (Sanhaçu-cinzento) que ocorrendo em áreas antrópicas, jardins e bordas de mata e Conirostrum bicolor (Figurinha-do-rabocastanho), ocorrendo em áreas de matas abertas e matas mesófilas. A família compreende um grupo de aves onívoras e seu forrageamento ocorre em grupos (casais, grupos gregários e até mesmo bandos mistos), além de solitariamente. Suas principais fontes de alimento são frutos, néctar e insetos (SIGRIST, 2009).

A terceira mais abundante, com 8 espécies cada, foram as famílias throchilidae e picidae. Os throchilidae representam o grupo numericamente e ecologicamente dominante nas interações aves-flores na região Neotropical, onde desempenham um importante papel na polinização de diversas angiospermas, pois possuem hábitos nectarívoros e insetívores, apresentando fácil adaptação em áreas antropizadas (MENDONÇA et al., 2005).

A família picidae é a família dos pica-paus. Possui hábitos alimentar predominantemente insetívoro, alimentam-se de larvas, frutos, sementes e mel. Caçam insetos, especialmente sob a casca. Atacam ninhos de marimbondos e vespas. Vivem em áreas campestres, pastos, eucaliptais, capoeiras ralas, buritizais, plantações, áreas rurais e bordas de mata. Vive também em cidades, parques, jardins, pomares, bordas de brejos arborizados, vive solitário ou aos pares dependendo da espécie (SICK, 2001). A espécie mais abundante dessa família foi a Melanerpes candidus (Pica-pau-branco), sempre avistado em áreas de campo sujo e bordas de mata.

As famílias columbidae e emberezidae apresentaram 7 espécies cada uma. As pombas e os pardais representantes respectivos a cada uma das famílias também possuem hábitos associados a áreas antropizadas, campos e bordas de mata. A família accipitridae apresentou 6 espécies, tratam-se de predadores presentes em todo tipo de habitat. Assim, baseado na riqueza de espécies acima, houve o predomínio de espécies generalistas, das quais, estão amplamente associadas à ocupação humana como 
áreas urbanas, áreas rurais, bordas de mata e áreas de campo sujo. Tais espécies representam 47,6\% do total de espécies observadas.

As outras espécies que se distribuem pelas unidades amostrais, são representantes das famílias: ardeidae, cuculidae, falconidae, furnaridae, hirundinidae, icteridae, pavonidae, piridae, psitacidae, rallidae e turdidae, não havendo grande variação na diversidade de espécies encontradas entre os fragmentos florestais, representam espécies com hábitos específicos de ambientas aquáticos, interiores de mata, matas mesófilas e dossel florestal.

Estudos afirmam que espécies de generalistas são ávidas consumidoras de grãos e frutas em regiões agrícolas tropicais e subtropicais e as características da paisagem são os fatores determinantes na flutuação populacional dessas espécies. O mesmo ocorre com espécies de aves que utilizam ambientes antropizados. São capazes de modificar seu comportamento de forrageio de acordo com as mudanças na constituição da paisagem (VILLARD et al., 1994; TRACEY et al., 2007; AHMAD et al., 2012).

\section{Análise da população de Avifauna}

A Tabela 3 apresenta os resultados das análises dos fragmentos de acordo com os Índices de Riqueza de Menhinick $\left(D_{M n}\right)$ e Shannon- Wiener $\left(H^{\prime}\right)$. O fragmento 15 foi o que apresentou o maior valor de $D_{M n}(3,20)$ e $\mathrm{H}^{\prime}$ de $(3,81)$ entre as unidades amostradas. Por outro lado o fragmento de menor riqueza e diversidade foi o fragmento 4, com valores de $D_{M n}$ de 1,50 e H' de 2,85 respectivamente. O $D_{M n}$ estima o número de espécies e o número de indivíduos de uma comunidade. $\mathrm{O} \mathrm{H}^{\prime}$ considera o número de espécies e a proporção de cada espécie em relação ao todo. Normalizando os dados e diminuindo a probabilidade de erro dos cálculos.

Tabela 3: Indicadores de riqueza, diversidade e abundância da avifauna.

\begin{tabular}{|c|c|c|c|c|}
\hline Fragmentos & IEA & Índice de Menhinick & Shannon-Wiener & Simpson \\
\hline 1 & 0,57 & 2,67 & 3,71 & 0,97 \\
\hline 2 & 0,60 & 2,40 & 3,41 & 0,96 \\
\hline 3 & 0,67 & 2,45 & 3,44 & 0,96 \\
\hline 4 & 0,54 & 1,50 & 2,85 & 0,92 \\
\hline 5 & 0,46 & 1,85 & 3,21 & 0,95 \\
\hline 6 & 0,60 & 2,12 & 3,19 & 0,94 \\
\hline 7 & 0,46 & 1,86 & 3,12 & 0,94 \\
\hline 8 & 0,69 & 1,58 & 3,14 & 0,94 \\
\hline 9 & 0,53 & 2,18 & 3,33 & 0,95 \\
\hline 10 & 0,65 & 2,07 & 3,51 & 0,96 \\
\hline 11 & 0,60 & 2,32 & 3,59 & 0,96 \\
\hline 12 & 0,67 & 2,61 & 3,75 & 0,97 \\
\hline 13 & 0,54 & 2,54 & 3,68 & 0,96 \\
\hline 14 & 0,61 & 2,57 & 3,63 & 0,97 \\
\hline 15 & 0,62 & 3,20 & 3,81 & 0,97 \\
\hline
\end{tabular}

De acordo com o índice de similaridade de Simpson, todos os fragmentos possuem uma baixa diversidade, devido seus valores estarem próximos a 1, havendo uma variação pequena entre os fragmentos. Os valores de Simpson refletem a probabilidade de dois indivíduos escolhidos ao acaso na comunidade pertencerem à mesma espécie. Como os valores variam de $0 \mathrm{a} 1$ e quanto mais alto for, maior a probabilidade de os indivíduos serem da mesma espécie, ou seja, maior a dominância e menor a diversidade.

Quando o índice de diversidade diminui, indica que há competição interespecífica e que o local é menos diversificado, tendendo a dominância de uma determinada espécie, porém quando há um aumento 
deste índice, há um indicativo de que o local é bastante diversificado, mesmo que o número de indivíduos seja reduzido (RODRIGUES, 2010).

\section{Identificação das áreas de risco e ranking das áreas prioritárias para conservação da avifauna}

Com base na última equação, os fragmentos florestais foram ranqueados de acordo com o nível de perturbação e de fragilidade relacionados à pressão causada pelos diferentes tipos de uso da terra, conservação do fragmento e biodiversidade da avifauna. A tabela 4 mostra o ranking do nível de prioridade para conservação dos fragmentos. Sendo a coluna de Riscos o produto final da multiplicação entre IEA e os indicadores de diversidade, riqueza e abundância da avifauna. Indicando o ranking decrescente final de Risco entre os fragmentos.

Tabela 4: Ranking de Risco dos fragmentos avaliados.

\begin{tabular}{|c|c|c|c|c|c|c|}
\hline Fragmentos & IEA & NDVI & Índice de Menhinick & Shannon- Wiener & Simpson & Risco \\
\hline $4^{\mathrm{B}}$ & 0,54 & 0,65 & 1,50 & 2,85 & 0,92 & 1,37 \\
\hline $7^{\mathrm{M}}$ & 0,46 & 0,68 & 1,86 & 3,12 & 0,94 & 1,69 \\
\hline $5^{M}$ & 0,46 & 0,70 & 1,85 & 3,21 & 0,95 & 1,83 \\
\hline $8^{M}$ & 0,69 & 0,63 & 1,58 & 3,14 & 0,94 & 2,05 \\
\hline $6^{M}$ & 0,60 & 0,63 & 2,12 & 3,19 & 0,94 & 2,40 \\
\hline $9^{M}$ & 0,53 & 0,68 & 2,18 & 3,33 & 0,95 & 2,50 \\
\hline $10^{\mathrm{M}}$ & 0,65 & 0,64 & 2,07 & 3,51 & 0,96 & 2,89 \\
\hline $2^{\mathrm{B}}$ & 0,60 & 0,68 & 2,40 & 3,41 & 0,96 & 3,19 \\
\hline $11^{\mathrm{M}}$ & 0,60 & 0,68 & 2,32 & 3,59 & 0,96 & 3,25 \\
\hline $13^{A}$ & 0,54 & 0,68 & 2,54 & 3,68 & 0,96 & 3,29 \\
\hline $14^{\mathrm{A}}$ & 0,61 & 0,66 & 2,57 & 3,63 & 0,97 & 3,65 \\
\hline $1^{\mathrm{B}}$ & 0,57 & 0,67 & 2,67 & 3,71 & 0,97 & 3,66 \\
\hline $3^{B}$ & 0,67 & 0,68 & 2,45 & 3,44 & 0,96 & 3,67 \\
\hline $12^{\mathrm{A}}$ & 0,67 & 0,67 & 2,61 & 3,75 & 0,97 & 4,29 \\
\hline $15^{A}$ & 0,62 & 0,68 & 3,20 & 3,81 & 0,97 & 4,97 \\
\hline
\end{tabular}

Legenda: ${ }^{\text {B }}$ - Fragmentos inseridos na subdivisão do Baixo Rio Una; ${ }^{\mathbf{M}}$ - Fragmentos inseridos na subdivisão do Médio Rio Una; ${ }^{\text {A }}$ - Fragmentos inseridos na subdivisão do Alto Rio Una.

O nível de risco dos fragmentos foram estabelecidos no intervalo de classes que variam de 0 a 5 . Portanto, quanto mais próximo de 0 maior o nível de risco e quanto mais próximo de 5 menor o risco para a biodiversidade dos fragmentos. De acordo com os valores de abundância, IEA e NDVI, como visto na Tabela 5, o nível de risco está diretamente relacionado à diversidade e riqueza das espécies da avifauna, bem como ao nível de pressão antrópica exercida no entorno dos fragmentos. A figura 6 apresenta o Mapa de risco e fragilidade da avifauna.

Segundo o cálculo dos indicadores, os fragmentos 12 e 15 foram classificados como sendo de Baixo Risco. Estas áreas estão diretamente ligadas ao predomínio de áreas de matas, pastagem e campos sujos no seu entorno, esses tipos de uso do solo possuem característica ecológica de permeabilidade, permitindo o fluxo das espécies da avifauna entre os fragmentos. Portanto, podem ser classificadas ecologicamente como áreas estáveis ou de baixa prioridade para conservação. Esses fragmentos estão inseridos na região morfológica do Alto Una, região da cabeceira da bacia do Rio Una, com predomínio de áreas verdes, pasto e grande conectividade entre fragmentos florestais.

As áreas classificadas como de risco intermediário ou Médio Risco foram os fragmentos 1, 2, 3, 6, 8, 9, 10,11,13 e 14. Os fragmentos 1, 2 e 3 estão inseridos na região morfológica do Baixo Una, região caracterizada, de acordo com o mapeamento do uso do solo, pela proximidade do centro urbano no 
município de Ibiúna, com área de expansão urbana, com grande influência de campos sujos e solos expostos, oferecendo pressão aos fragmentos, além da interferência nos hábitos de forrageamento da avifauna.

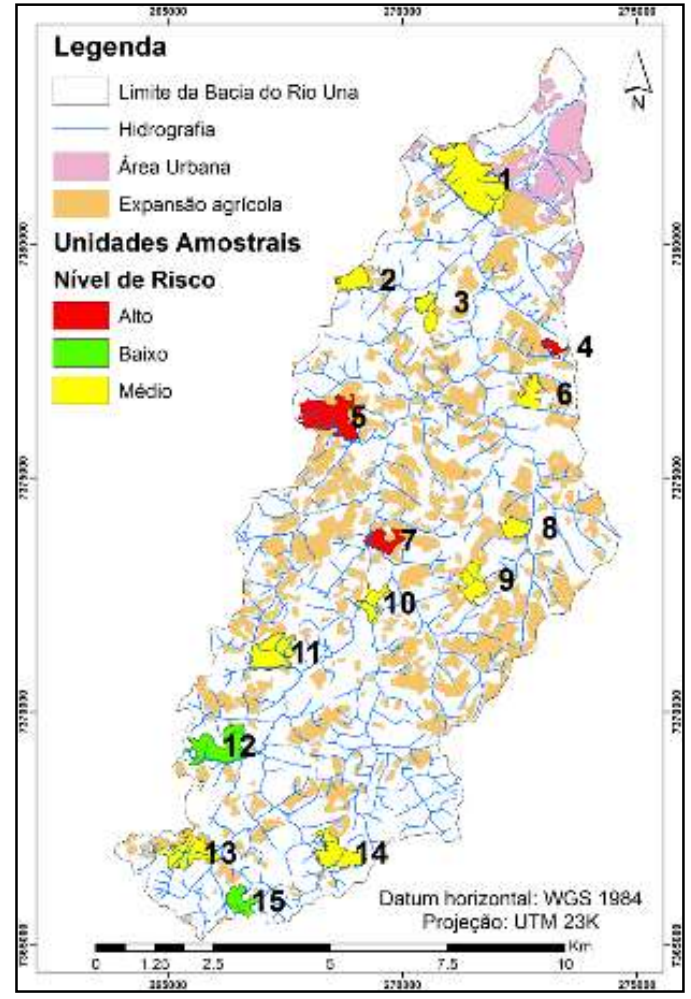

Figura 6: Mapa de Risco e Fragilidade da avifauna.

Os fragmentos 6, 8, 9, 10 e 11 estão inseridos na região morfológica do Médio Una, região predominada por agriculturas de curto e longo período com poucas áreas de pastagem. Sendo os fragmentos 6 e 8 os de menor qualidade vegetal entre todos os fragmentos da bacia, entretanto, eles possuem alta biodiversidade e abundancia da avifauna comparado a outros fragmentos, além de possuírem uma das áreas com menor influência da ação antrópica externa, sendo o fragmento 8 o melhor IEA de toda a bacia hidrográfica, no entanto a sua baixa abundancia de espécies da avifauna, tornam a classificação final como áreas de Média prioridade de conservação, ou parcialmente estáveis.

Com relação às áreas de Alto Risco ou de maior fragilidade, foram identificadas as unidades amostrais 4, 5 e 7 apresentam maior Risco de perda da biodiversidade da avifauna. Esses fragmentos possuem baixa qualidade vegetal, baixa biodiversidade além de sofrer grande influência da pressão antrópica. 0 entorno dessas áreas é composto por áreas de agricultura de curto período, pastagens e edificações rurais, o que podem acelerar o processo de perda da biodiversidade, tanto animal quanto vegetal. Portanto, esses fragmentos possuem alta prioridade de conservação, podendo ser classificado como instáveis, do ponto de vista ecológico.

De forma geral, subentende-se que as áreas de Médio e Alto risco sofrem interferência das áreas agrícolas no que tange ao comportamento de forrageamento das espécies da avifauna. Aumentando a oferta de alimento nos campos de plantação, tanto de cereais e grãos como de outros animais que são atraídos pelos mesmos fins. Portanto, a grande diversidade de espécies pode ser atribuída à conectividade, ou a 
proximidade entre outros fragmentos florestais. Favorecendo a conectividade e a continuidade do fluxo gênico entre as florestas.

Como exemplo, pode-se observar os fragmentos classificados com baixo risco do Alto Una, pois demonstram que as áreas de pastagens efetivamente exercem menor interferência na perda da biodiversidade do que as áreas de agricultura. A região tem predomínio de áreas de mata e pastagens. Demonstrando que as áreas de pastagens efetivamente exercem menor interferência na biodiversidade do que as áreas de agricultura. Em uma abordagem geral, os fragmentos com maior perímetro, associados às áreas rurais, são os de menor prioridade para preservação. As áreas de Alto risco são os fragmentos próximo às zonas urbanas, áreas de expansão agrícola e áreas de pastagens.

\section{CONCLUSÕES}

A realização de pesquisas e desenvolvimento de metodologias envolvendo a resposta de populações e comunidades de aves brasileiras, sob as ações da urbanização e aos processos que deterioram os seus habitats, fornecem inferências importantes para a avaliação das mudanças associadas ao desenvolvimento humano em contraste com os ambientes. Corroborando com esse fato, foi possível observar diferentes comportamentos das populações de avifauna referente ao potencial de interferências da pressão antrópica nos ecossistemas. Nesse sentido, nota-se a importância da manutenção de áreas verdes com importante papel na conservação da avifauna, uma vez que são refúgios ou 'ilhas' para as espécies que, pressionadas pela degradação ambiental das áreas naturais se ajustam às diferentes intervenções humanas.

A utilização do IEA, somado aos indicadores da biodiversidade pode ser um instrumento de uso intenso por gestores ambientais na identificação das áreas que oferecem risco a biodiversidade e áreas prioritárias para conservação. Uma vez que pode auxiliar na compreensão do comportamento de espécies ameaçadas e na elaboração de propostas de mitigação de grandes impactos ambientais. Além disso, é uma metodologia bastante útil para auxiliar na gestão e planejamento de políticas públicas voltadas para o meio ambiente. Os resultados obtidos servem para a definição de áreas específicas voltadas à conservação da vegetação e ampliação de as áreas de mata ciliar, consequentemente promovem a conectividade entre os fragmentos, o que assegura e manutenção da diversidade biológica tanto da fauna quanto da flora.

\section{REFERÊNCIAS}

ABREU, C. I. V.; ZAMPIERON, S. L. M.. Perfil da Fauna de Hymenoptera Parasítica em um Fragmento de Cerrado pertencente ao Parque Nacional da Serra da Canastra (MG), a partir de duas Armadilhas de Captura. Ciência et Praxis v.3, n.1, p.61-68, 2009

AHMAD, S.; KHAN, H. A.; JAVED, M.; REHMAN, K. U.. Management of maize and sunflower against the depredations of Rose-ringed Parakeet (Psittacula krameri) using mechanical repellents in an agro-ecosystem. International Journal of Agriculture and Biology, v.14, n.1, p.286-290, 2012.

ALEIXO, A.; POLETTO, F.. Birds of an open vegetation enclave in southern Brazilian Amazonia. The Wilson Journal of
Ornithology, v.119, n.4, p.610-630, 2007. DOI:

http://doi.org/10.1676/06-153.1

AMBUEL, B.; TEMPLE S. A.. Area-Dependent Changes in the Bird Communities and Vegetation of Southern Wisconsin Forests. Ecology, v.64, n.1, p.1057-1068, 1983.

BARROS, R. S. M.. Medidas de diversidade biológica. Juiz de Fora. UFJF, 2007.

BECKER, M.; DALPONTE, J. C.. Rastros de Mamíferos Silvestres Brasileiros: Um guia de Campo. 2 ed. Brasília: Technical Books, 1991. 
BUREL, F.; BAUDRAY, J.. Ecologia del Paisaje: Conceptos, Métodos y aplicaciones. Madrid: Mundi-Prensa, 2002.

CANALE, C. A.; PERES, C. A.; GUIDORIZZI, C. E.; GATTO, C. A. F.; KIERULFF, M. C. M.. Pervasive defaunation of forest remnants in a tropical biodiversity hotspot. PLoS ONE, v.7, n.8, p.1-9, 2012. DOI:

http://doi.org/10.1371/journal.pone.0041671

CARDOSO, C. A. L.; FARIA, F. S. R.. O uso do geoprocessamento na análise ambiental como subsídio para a indicação de áreas favoráveis a criação de unidades de conservação para o uso sustentável do Minhocuçu Rhinodrilus alatus. E-Scientia, v.3, n.1, p.1-26, 2010.

CARDOSO, C. A.; DIAS, H. C. T.; SOARES, C. P. B.; MARTINS, S V.. Caracterização morfométrica da bacia hidrográfica do rio Debossan, Nova Friburgo, RJ. Revista Árvore, v.30, n.1, p.241-248, 2006.

CARVALHO JÚNIOR, O.; LUZ, N. C.. Pegadas: Série Boas Práticas. 3 ed. Belém: EDUFPA, 2008.

CAVANI, A. C. M.; ALBUQUERQUE-FILHO, J. L.. Plano de Bacias, Mapa Diagnóstico. São Paulo: IPT, 2008.

CORRÊA, B. S.; PASSAMANI, M.; MOURA, A. S.. Avaliação do efeito borda na distribuição da avifauna em fragmentos florestais de Cerrado. Revista Agroambiental. v.3, n.1. p.3741, 2011. DOI: http://doi.org/10.18406/2316$\underline{1817 v 3 n 32011359}$

DÁRIO, F. R.; ALMEIDA, A. F.. Influência de corredor florestal sobre a avifauna da Mata Atlântica. Scientia Florestalis, v.58, n.1, p.99-109, 2002.

DÁRIO, F. R.; VINCENZO, M. C. V.; ALMEIDA, A. F.. Avifauna em fragmentos da Mata Atlântica. Ciência Rural, v.32, n.1, p.989-996, 2002.

DEVELEY, P. F.; MARTENSEN, A. C.. As aves da Reserva Florestal do Morro Grande (Cotia - SP). Biota Neotropica, v.6, n.1, p.1-16, 2006.

ESRI. Environmental Systems Research Institute. ArcGIS 10: Getting started with ArcGIS. Redlands: ESRI, 2010.

FERGER, S. W.; SCHLEUNING, M.; HEMP, A.; HOWELL, K. M.; BOHNINH-GAESE, K.. Food resources and vegetation structure mediate climatic effects on species richness of birds. Global Ecology and Biogeography, v.23, n.5, p.541549, 2014. DOI: http://doi.org/10.1111/geb.12151

FORMAN, R. T. T.; GODRON, M.. Landscape Ecology Literature Review. Canada: Silva Ecosystem Consultants Ltda, 1992.

LIAO, J.; LI, Z.; HIEBELER, D. E.; EL-BANA, M.; DECKMYN, G.; NIJS, I.. Modelling plant population size and extinction thresholds from habitat loss and habitat fragmentation: Effects of neighbouring competition and dispersal strategy. Ecological Modelling. v.268, n.1, p.9-17, 2013. DOI: http://doi.org/10.1016/j.ecolmodel.2013.07.021

LOURENÇO, R. W.; SILVA, D. C. C.; MARTINS, A. C. G.; SALES, J. C. A.; ROVEDA. S. R. M. M.; ROVEDA, J. A. F.. Use of fuzzy systems in the elaboration of an anthropic pressure indicator to evaluate the remaining forest fragments. Environmental
Earth Sciences, v.73, n.3, p.2481-2488, 2015. DOI: http://doi.org/10.1007/s12665-015-4253-6

LOURENÇO, R. W.; SILVA, D. C. C.; SALES, J. C. A.. Development of a methodology for evaluation of the remaining forest fragments as a management tool and environmental planning. Ambiência, v.10, n.3, p.685-698, 2014.

LOURENÇO, R. W.; SILVA, D. C. C.; SALES, J. C. A.. Geoprocessamento como ferramenta de gestão e planejamento ambiental: $O$ caso da cobertura vegetal em áreas urbanas. In: SMITH, W. S.; MOTA JÚNIOR, V.; LIMA, J. C.. Biodiversidade do Município de Sorocaba. Sorocaba: Secretaria Municipal de Meio Ambiente, 2014. p.65-78.

MARENZI, R. C.; RODERJAN, C. V.. Estrutura Espacial da Paisagem da Morraria da Praia Vermelha (SC): Subsídio à Ecologia da Paisagem. Floresta, v.35, n.2, p.259-269, 2005. DOI: http://doi.org/10.5380/rf.v35i2.4614

MENDONÇA, L. B.; ANJOS, L.. Beija-flores (Aves, Trochilidae) e seus recursos florais em uma área urbana do Sul do Brasil. Revista Brasileira de Zoologia, v.22, n.1, p.51-59, 2005. DOI: http://doi.org/10.1590/S0101-81752005000100007

NIEMI, G. J.; MCDONALD, M. E.. Application of ecological indicators. Annual Review of Ecology, Evolution and Systematics, v.35, n.1, p.89-111, 2004. DOI: http://doi.org/10.1146/annurev.ecosys.35.112202.130132

PENSE, M. R.; CARVALHO, A. P. C.. Biodiversidade de aves do Parque Estadual do Jaraguá (SP). ConScientiae Saúde, v.4, n.1, p.55-62, 2005.

PIRATELLI, A.; SOUSA, S. D.; CORRÊA, J. S.; ANDRADE, V. A.; RIBEIRO, R. Y.; AVELAR, L. H.; OLIVEIRA, E. F.. Searching for bioindicators of forest fragmentation: passerine birds in the Atlantic forest of southeastern Brazil. Brazilian Journal of Biology, v.68, n.1, p.259-268, 2008.

RODRIGUES, W. C.. Estatística Aplicada. 8 ed. Rio de Janeiro: Creative Commons Licence, 2010.

SALES, J. C. A.; SILVA, D. C. C.; ROMAGNANO, L, F. T.; BERTAGNA, R.; LOURENÇO, R. W.. Avaliação do Impacto Ambiental causado pelas alterações espaço temporal do uso do solo e da cobertura vegetal utilizando o modelo das cadeias de Markov. Ciência e Natura, v.38, n.1, p.115-124, 2016. DOI: http://doi.org/10.5902/2179-460X18641

SANTOS, J. T. S.; PENA, H. W. A.. Geoprocessamento aplicado a ecologia de paisagem: uma análise da dinâmica da llha do Papagaio - PA, Amazônia-Brasil. Revista OIDLES, v.5, n.1, p.116, 2011.

SICK, H.. Ornitologia Brasileira. 3 ed. Rio de Janeiro: Nova Fronteira, 2001.

SIGRIST, T.. Avifauna Brasileira. 4 ed. São Paulo: Avis Brasilis, 2009.

SIGRIST, T.. Guia de Campo Avis Brasilis: Avifauna Brasileira. 4 ed. São Paulo: Avis brasilis, 2013.

SILVA, D. C. C.; SALES, J. C. A.; ALBUQUERQUE-FILHO, J. L.; LOURENÇO, R. W.. Características morfométricas e suas implicações no acúmulo de sedimentos em reservatórios: o 
caso da Represa Hedberg, Iperó/SP. RA'E GA: o Espaço

Geográfico em Análise, v.36, n.1, p.225-245, 2016.

SILVEIRA NETO, S.; MONTEIRO, R. C.; ZUCCHI, R. A.; MORAES, R. C. B.. Uso da análise faunística de insetos na avaliação do impacto ambiental. Scientia Agricola, v.52, n.1, p.9-15, 1995.

SILVEIRA, L. F.; UEZU, A.. Checklist das aves do Estado de São Paulo, Brasil. Biota Neotropica, v.11, n.1, p.1-28, 2011.

TRACEY, J.; BOMFORD, M.; HART, Q.; SAUNDERS, G.; SINCLAIR, R.. Managing bird damage to fruit and other horticultural crops. Canberra: Bureau of Rural Sciences, 2007
VIANA V. M.; PINHEIRO L. A. V.. Conservação da biodiversidade em fragmentos florestais. SÉRIE TÉCNICA IPEF, v.12, n.32, p.25-42, 1998.

VILLARD, M.; TAYLOR, P. D.. Tolerance to habitat fragmentation influences the colonization of new habitat by forest birds. Oecologia, v.98, n.3, p.393-401, 1994. DOI: http://doi.org/10.1007/BF00324229

WATRIN, O. S.; VENTURIERI, A. I.. Métricas de paisagem na avaliação da dinâmica do uso da terra em projetos de assentamentos no Sudeste Paraense. In: SIMPÓSIO BRASILEIRO DE SENSORIAMENTO REMOTO, 12. Anais. Goiânia: INPE, 2005. 